\title{
Is conservative management noninferior to interventional treatment for moderate to large primary spontaneous pneumothoraces?
}

Herman Johal, MD*; Andrew McRae, $\mathrm{MD}^{+}$; Greg Beller, MD*

\section{INTRODUCTION}

Background: Current American and British Pulmonology Guidelines advise immediate interventional management of most large ( $>2 \mathrm{~cm}$ at hilum; $>3 \mathrm{~cm}$ at apex) primary spontaneous pneumothoraces. ${ }^{1,2}$

Objectives: Is observation of moderate-to-large primary spontaneous pneumothoraces $(>32 \%$ by Collins method) noninferior to immediate interventional management assessed by complete lung re-expansion within 8 weeks.
\end{abstract}

\section{METHODS}

\section{Design}

Multicenter randomized, open-label, noninferiority trial, with $9 \%$ noninferiority margin to demonstrate conservative management is not inferior to interventional treatment.

\section{Setting}

Thirty-nine hospitals in Australia and New Zealand.

\section{Subjects}

Patients aged 14-50 years with first-time unilateral primary spontaneous pneumothorax of $\geq 32 \%$.

\section{Intervention}

Conservative (repeat imaging after 4 hours, discharge if no supplemental oxygen required; chest tube inserted if clinically unwell) v. intervention (chest tube $(\leq 12 \mathrm{~F})$ with suction; remove and discharge if resolved on chest $\mathrm{x}$-ray at 4 hours or admission).

\section{Outcomes}

The primary noninferiority outcome was complete radiographic resolution of the pneumothorax 8 weeks after randomization. Numerous secondary outcomes.

\section{RESULTS}

A total of 316 of the 2,637 screened patients underwent randomization; 154 patients to the intervention group, and 162 in the conservative-management group. The prespecified noninferiority margin was (-9\%) for re-expansion by 8 weeks. The authors based this on an expected resolution rate of $99 \%$ for the intervention group and believed a failure rate of 1 in 10 would be clinically acceptable. A margin this size may bias toward finding statistical noninferiority where there is clinical inferiority.

In the Complete Case analysis, the resolution rate in the intervention group was 129/131 (98.5\%). Five patients were lost to follow-up, and 18 did not have chest radiography data available at 8 weeks. In the conservative group, 118/125 (94.4\%) had a resolved pneumothorax on chest radiography at 8 weeks, 3 patients were lost to follow-up, and 34 did not have complete data. The Risk Difference was $-4.1 \%$, 95\% CI -8.6 to $0.5, p=0.02$.

From the ${ }^{*}$ CCFP-Emergency Medicine Residency Program, University of Calgary, Calgary, AB; and the ${ }^{\dagger}$ Department of Emergency Medicine, Cumming School of Medicine, University of Calgary, Calgary, AB.

Correspondence to: Dr. Herman Johal, Department of Emergency Medicine, 140329 Street NW, Calgary, AB T2N 2T9; Email: h.johal03@gmail.com

(C) Canadian Association of Emergency Physicians 2020

CJEM 2020;22(6):772-773

DOI 10.1017/cem.2020.419 


\section{APPRAISAL}

\section{Strengths}

- First study to assess conservative v. interventional therapy in moderate-large primary pneumothoraces

- Used both clinician and patient-centered secondary outcomes that can impact a shared decision-making model

- In addition to a complete case analysis, they also performed two sensitivity analyses, including one in which missing data were imputed as treatment failure (i.e. worst-case scenario).

\section{Limitations}

- This study was unblinded, which could bias findings (treating physicians were more likely to report radiographic resolution of pneumothorax in the intervention group when compared with blinded radiologists)

- The two sensitivity analyses identify potential statistical fragility in the main findings

- A shorter-term primary outcome might reveal greater disparity between conservative v. treatment groups

- The noninferiority margin of $9 \%$ is large

- $15.4 \%$ of patients (25) in the conservative treatment group required intervention

- $19 \%$ of randomized patients (60) lost to follow-up or had missing outcome data

- Radiographic resolution is not a patient-centered outcome.

\section{CONTEXT}

Conservative management has been the standard of care for small primary spontaneous pneumothoraces for many years. The British Thoracic Society ${ }^{1}$ and the American College of Chest Physicians ${ }^{2}$ both currently recommend that clinically stable patients with large primary spontaneous pneumothorax undergo interventional management with a small-bore catheter or chest tube, followed by hospitalization in many cases. In Canada, these patients are commonly treated with a pigtail catheter and discharged home from the emergency department (ED). This study is a step toward validating an observational approach to large pneumothoraces in a carefully selected population.

\section{BOTTOM LINE}

Although this study contains some statistical fragility, there is modest evidence that a conservative approach may be noninferior to intervention in carefully selected patients with first time, moderate to large spontaneous pneumothorax. While many Canadian ED physicians may choose a pigtail catheter, any form of tube thoracostomy has potential adverse consequences. This study may identify an alternative option that can be used in shared decision-making with the appropriately selected patient.

Keywords: Emergency medicine, respiratory medicine, research: patient outcomes

Competing interests: None declared.

\section{REFERENCES}

1. MacDuff A. 2010 BTS Pleural Disease Guideline Group. Management of spontaneous pneumothorax: British Thoracic Society Pleural Disease Guideline. Thorax 2010;65(Suppl 2): ii18-31.

2. Baumann MH, Strange C, Heffner JE, et al. Management of spontaneous pneumothorax: an American College of Chest Physicians Delphi Consensus Statement. Chest 2001;119 (2):590-602. 